Mathematical Problems in Mechanics

\title{
Penalized Direct Forcing and Projection Schemes for Navier-Stokes
}

\author{
Michel Belliard ${ }^{\mathrm{b}}$ Clarisse Fournier ${ }^{\mathrm{c}}$ \\ CEA, Département d'Etudes des Réacteurs, DEN/DER/SSTH \\ ${ }^{\mathrm{b}}$ CEA, DEN, DER/SSTH/LDAL - Cadarache, F-13108 St Paul-lez-Durance, France \\ ${ }^{\mathrm{c}}$ CEA, DEN, DER/SSTH/LDAL, F-38054 Grenoble, France \\ Received $* * * * ;$ accepted after revision +++++ \\ Presented by
}

\begin{abstract}
This note presents a new method of direct forcing to deal with obstacles in incompressible flows. It mixes projection schemes and velocity $L^{2}$ penalty schemes. The penalized direct forcing term is distributed in the velocity prediction and the correction equations. It leads to a natural treatment in the correction equation of the boundary conditions in pressure around obstacles. A numerical experiment provided an illustration of the method. To cite this article: M. Belliard, C. R. Acad. Sci. Paris, Ser. I 340 (2005).

\section{Résumé}

Méthode de Forçage Direct Pénalisé et Schémas de Projection pour Navier-Stokes. Cette note présente une nouvelle méthode de forçage directe pour prendre en compte des obstacles dans un écoulement incompressible. Elle mélange méthodes de projection et de pénalisation $L^{2}$ des vitesses. La ventilation du terme de forçage direct pénalisé dans les équations de prédiction et de correction conduit à un traitement naturel des conditions aux limites pour la correction de pression aux bords des obstacles. Une expérience numérique est présentée à titre d'illustration. Pour citer cet article : M. Belliard, C. R. Acad. Sci. Paris, Ser. I 340 (2005).
\end{abstract}

\section{Introduction}

The well-known incompressible Navier-Stokes equations allow the description of the behavior of incompressible flows ( $\operatorname{div} \mathbf{u}=0$ with $\mathbf{u}$ the fluid velocity). Mass and momentum balance equations in the fluid domain $\Omega_{f}$ read as (with Dirichlet boundary conditions -BC -, $\mathbf{u}=\mathbf{u}_{D}$ on $\partial \Omega_{f}$ ):

\footnotetext{
Email addresses: michel.belliard@cea.fr (Michel Belliard), clarisse.fournier@cea.fr (Clarisse Fournier).
} 


$$
\left\{\begin{array}{ccc}
\partial_{t} \mathbf{u}+\mathbf{u} \otimes \nabla \mathbf{u}+\rho^{-1} \nabla p-\operatorname{div}(\nu \nabla \mathbf{u}) & =\mathbf{f} & \text { in } \Omega_{f} \\
\operatorname{div} \mathbf{u} & =0 & \text { in } \Omega_{f}
\end{array}\right.
$$

with $p$ the pressure, $\rho$ the (constant) density, $\nu$ the cinematic viscosity and $\mathbf{f}$ the volume force.

The projection method (or fractional step method) for an incompressible fluid was introduced by Chorin and Temam in $1968[2,10]$. The incremental projection scheme of Shen $[9]$ reads as follow. ${ }^{1}$ First, a non divergence free velocity $\widetilde{\mathbf{u}}$ is predicted using the previous time step pressure. For example, a linearized implicit Euler time discretization ${ }^{2}$, with $\mathbf{u}^{n, *}$ given by an Adam-Bashforth scheme, leads to:

$$
\frac{\widetilde{\mathbf{u}}-\mathbf{u}^{n}}{\Delta t}+\mathbf{u}^{n, *} \otimes \nabla \widetilde{\mathbf{u}}+\rho^{-1} \nabla p^{n}-\operatorname{div}(\nu \nabla \widetilde{\mathbf{u}})=\mathbf{f}^{n+1} \quad \text { in } \Omega_{f} \quad \text { and } \widetilde{\mathbf{u}}=\mathbf{u}_{D} \quad \text { on } \partial \Omega_{f} .
$$

Second, with the hypothesis $\operatorname{div}(\nu \nabla \widetilde{\mathbf{u}}) \sim \operatorname{div}\left(\nu \nabla \mathbf{u}^{n+1}\right)$ and $\mathbf{u}^{n, *} \otimes \nabla \widetilde{\mathbf{u}} \sim \mathbf{u}^{n, *} \otimes \nabla \mathbf{u}^{n+1}$, a correction stage is performed to recover the divergence free velocity $\mathbf{u}^{n+1}$ :

$$
\left\{\begin{array}{l}
\frac{\mathbf{u}^{n+1}-\widetilde{\mathbf{u}}}{\Delta t}=-\rho^{-1} \nabla \phi^{n+1} \quad \text { in } \Omega_{f} \quad \text { and } \quad \mathbf{u}^{n+1}=\mathbf{u}_{D} \quad \text { on } \partial \Omega_{f} \\
\operatorname{div} \mathbf{u}^{n+1}=\quad 0 \quad \text { in } \Omega_{f}
\end{array}\right.
$$

with $\phi^{n+1}=p^{n+1}-p^{n}$ the pressure correction. Considering the divergence free condition, we are able to compute $\phi^{n+1}$ and so on the divergence free velocity $\mathbf{u}^{n+1}$ and the new pressure $p^{n+1}$ by:

$$
\operatorname{div} \nabla \phi^{n+1}=\frac{\rho}{\Delta t} \operatorname{div} \widetilde{\mathbf{u}} \quad \text { in } \Omega_{f} \text { and } \nabla \phi^{n+1} \cdot \mathbf{n}=0 \quad \text { on } \partial \Omega_{f} .
$$

\section{Direct Forcing}

In the context of the Fictitious Domain methods, the Immersed Boundary Method was introduced by Peskin at the beginning of 1970 [8]. Considering obstacles in a flow, the fluid domain $\Omega_{f}$ is extended to $\Omega$ including the obstacle domain $\Omega_{s}: \Omega=\Omega_{f} \cup \Omega_{s}$. A body force is added to the momentum equation to take into account the obstacle. In 1997 Mohd-Yusof introduces the Direct Forcing method [7]:

$$
\frac{\mathbf{u}^{n+1}-\mathbf{u}^{n}}{\Delta t}=\mathbf{T}_{n, n+1}+\mathbf{F}_{n, n+1} \text { in } \Omega
$$

with here $\mathbf{T}_{n, n+1}=\mathbf{f}^{n+1}-\mathbf{u}^{n, *} \otimes \nabla \mathbf{u}^{n+1}-\rho^{-1} \nabla p^{n+1}+\operatorname{div}\left(\nu \nabla \mathbf{u}^{n+1}\right)$. The forcing term is defined as:

$$
\mathbf{F}_{n, n+1}=\chi_{s}\left[\frac{\left(\mathbf{v}_{s}^{n+1}-\mathbf{u}^{n}\right)}{\Delta t}-\mathbf{T}_{n, n+1}\right]
$$

with $\mathbf{v}_{s}$ the imposed fluid velocity around/inside the obstacle and $\chi_{s}: R^{3} \rightarrow R$ the discrete characteristic function or the volume ratio function of the obstacle, see [3].

Remark 1 The imposed velocity $\mathbf{v}_{s}$ needs to be interpolated from the fluid velocity field $\widetilde{\mathbf{u}}$ and the boundary condition to improve the accuracy $\left(\mathbf{v}_{s}=\bar{\varepsilon} \widetilde{\mathbf{u}}\right.$, see Fadlun et al. [3]).

Remark 2 The direct forcing should be taken into account into the projection equation (4) through the interpolation matrix $\bar{\varepsilon}\left(\operatorname{div} \bar{\varepsilon} \nabla \phi=\frac{\rho}{\Delta t}\right.$ div $\left.\widetilde{\mathbf{u}}\right)$ to get a free divergence velocity respecting the boundary conditions (consistent scheme, see Ikeno et al. [5]).

\footnotetext{
1 Projection schemes for dilatable or barotropic fluid can be found in $[4,6]$.

2 Using the Crank-Nicholson time scheme should be worth, but it isn't the goal of this Note.
} 


\section{Penalized Direct Forcing}

In this new method, the following forcing term is added to the right hand side of Navier-Stokes equations:

$$
\mathbf{F}_{n+1}=\frac{\chi_{s}}{\eta \Delta t}\left(\mathbf{v}_{s}^{n+1}-\mathbf{u}^{n+1}\right) \text { with } 0<\eta \ll 1 .
$$

It can be viewed as an implicit forcing term $c \frac{\chi_{s}}{\Delta t}\left(\mathbf{v}_{s}-\mathbf{u}\right)$ as well as a velocity $L^{2}$ penalty term $c \frac{\chi_{s}}{\eta}\left(\mathbf{v}_{s}-\right.$ $\mathbf{u}$ ) [1]. As for the Direct Forcing, interpolations can be used to determine the imposed velocity $\mathbf{v}_{s}$. In the following, $\mathbf{u}^{n+1}$ is the solution of penalized Navier-Stokes. Lets define $\mathbf{u}_{\eta}^{n+1}:=\lim _{\eta \rightarrow 0} \mathbf{u}^{n+1}$. Then, we have $\mathbf{u}_{\eta}^{n+1} \sim \mathbf{v}_{s}^{n+1}$. Following Angot et al. [1], $\mathbf{u}_{\eta}^{n+1}$ converges toward the N.-S. body fitted solution in $L^{2}\left(\Omega_{s}\right)$ norm with order $\leq 3 / 4$ in $\eta$. The new feature of our algorithm is that the forcing term (7) is distributed in the prediction and the correction stages of the projection leading to a natural consistent scheme:

$$
\left\{\begin{aligned}
\frac{\widetilde{\mathbf{u}}-\mathbf{u}^{n}}{\Delta t}+\mathbf{u}^{n, *} \otimes \nabla \widetilde{\mathbf{u}}+\rho^{-1} \nabla p^{n}-\operatorname{div} \nu \nabla \widetilde{\mathbf{u}} & =\mathbf{f}^{n+1}+\frac{\chi_{s}}{\eta \Delta t}\left(\mathbf{v}_{s}^{n+1}-\widetilde{\mathbf{u}}\right) \quad \text { in } \Omega \\
\frac{\mathbf{u}^{n+1}-\widetilde{\mathbf{u}}}{\Delta t} & =-\rho^{-1} \nabla \phi^{n+1}+\frac{\chi_{s}}{\eta \Delta t}\left(\widetilde{\mathbf{u}}-\mathbf{u}^{n+1}\right) \quad \text { in } \Omega
\end{aligned}\right.
$$

The first equation of (8) leads to the penalized predicted velocity: $\widetilde{\mathbf{u}}_{\eta}:=\lim _{\eta \rightarrow 0} \widetilde{\mathbf{u}} \sim \mathbf{v}_{s}^{n+1}$ in $\Omega_{s}$. Hence the Dirichlet boundary conditions of (2) are locally enforced on $\partial \Omega_{f} \cap \partial \Omega_{s}$. The second equation of (8) suggests:

$$
\frac{\mathbf{u}^{n+1}-\widetilde{\mathbf{u}}}{\Delta t}=-\check{\rho}^{-1} \nabla \phi^{n+1} \quad \text { in } \Omega \text { with suitable } \mathrm{BC} \text { on } \partial \Omega .
$$

This equation is similar to the first one of (3) with $\check{\rho}:=\rho\left(1+\frac{\chi_{s}}{\eta}\right)$ justifying the introduction of the term $\Delta t$ in (7). For $\chi_{s}=0$, we have $\check{\rho}=\rho$ and $\lim _{\eta \rightarrow 0} \check{\rho}(\eta) \approx \mathcal{O}\left(\eta^{-1}\right)$ for $\chi_{s}>0$. Using div $\mathbf{u}^{n+1}=0$ in $\Omega_{f}$ and $\operatorname{div} \mathbf{u}^{n+1}=\operatorname{div} \mathbf{v}_{s}$ in $\Omega_{s}$, instead of the second equation of (3), we get a slightly modification of the first equation of (4):

$$
\operatorname{div} \frac{\eta}{\eta+\chi_{s}} \nabla \phi^{n+1}=\frac{\rho}{\Delta t}\left(\operatorname{div} \widetilde{u}-\chi_{s} \operatorname{div} \mathbf{v}_{s}^{n+1}\right) \quad \text { in } \Omega \text { with suitable BC on } \partial \Omega .
$$

Lets remark that, in case of solid rotations and/or translations, $\operatorname{div} \mathbf{v}_{s}=0$ in $\Omega_{s}$. For $\chi_{s}=1$, we have an effective diffusion coefficient $\frac{\eta}{1+\eta} \approx \mathcal{O}(\eta) \ll 1$. Then, the homogeneous Neumann boundary conditions of (4) are locally enforced on $\partial \Omega_{f} \cap \partial \Omega_{s}$ in a natural way. Finally, the correction equation (9) allows the computation of $\mathbf{u}^{n+1}$. We have $\mathbf{u}_{\eta}^{n+1} \sim \widetilde{\mathbf{u}}_{\eta} \sim \mathbf{v}_{s}^{n+1}$ in $\Omega_{s}$

Remark 3 In $\Omega_{s}$, all the diffusion coefficients may vanish leading to numerical problems solving (10). Then, adding a pressure $L^{2}$ penalty term can be a cure, leading to a different formulation of the pressure correction equation (where $\phi_{0}$ is a prescribed pressure correction):

$$
\operatorname{div} \mathcal{O}(\eta) \nabla \phi^{n+1}+\frac{1}{\eta}\left(\phi^{n+1}-\phi_{0}\right)=\frac{\rho}{\Delta t}\left(\operatorname{div} \widetilde{u}-\operatorname{div} \mathbf{v}_{s}^{n+1}\right) \quad \text { in } \Omega_{s}
$$

\section{Numerical experiments}

Fig. 1, left, shows a 2D Poiseuille flow in a square channel rotated of $45^{\circ}$ with regard to the Cartesian mesh. Immersed Boundaries define the solid walls (black lines). Imposed near-wall velocities are linearly 

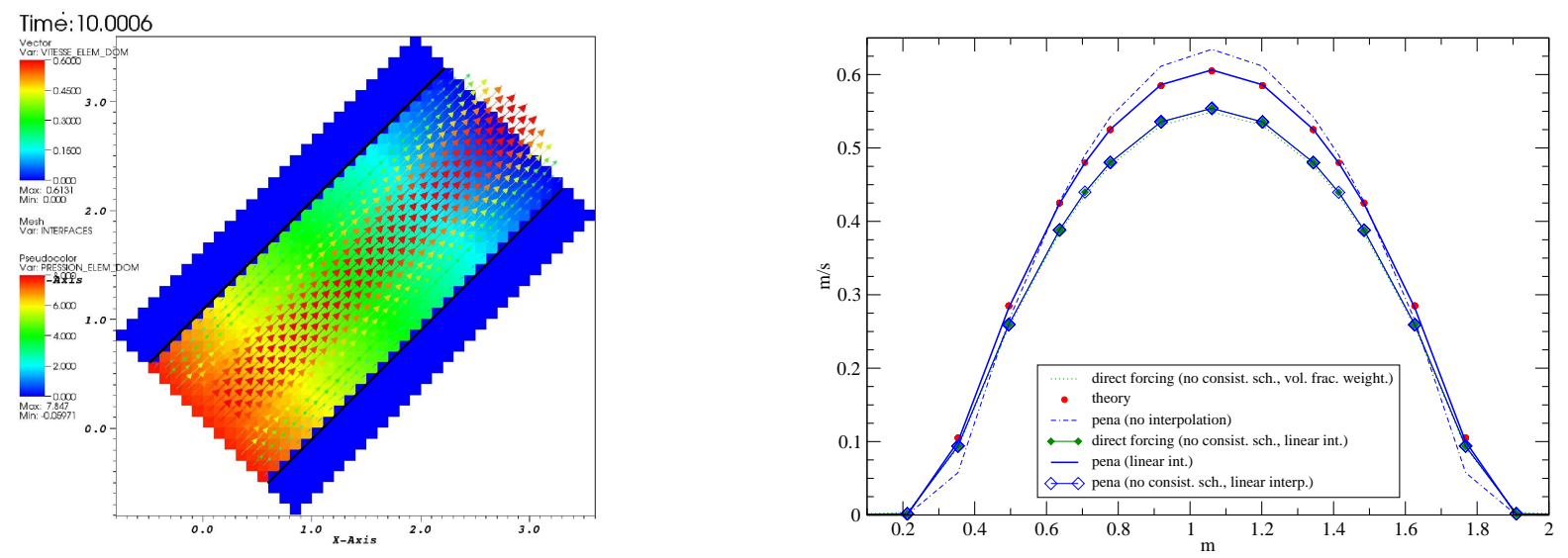

Figure 1. Poiseuille Flow: Direct Forcing and Penalized Direct Forcing. Left: Velocity and pressure fields (linearly interpolated Penalized Direct Forcing). Right: Velocity profiles (no interpolation or linear interpolation, no consistent or consistent scheme).

interpolated from the nearest free fluid velocities and the boundary conditions $\mathbf{v}_{s}^{n+1}=\bar{\varepsilon} \mathbf{u}^{n}$. Computations are done using the CEA CFD code Trio_U [11]. The value of the penalty coefficient $\eta$ is $10^{-12}$. Velocity profiles, obtained by the linearly interpolated Penalized Direct Forcing method, compare very well to the theoretical ones, see Fig. 1, right. Without consistent schemes the theoretical velocities are missed whatever the interpolation is. Moreover, the numerical order of the method remains about 2 in $L^{2}$ norm when using a second order discretization for the spatial operators.

\section{References}

[1] Ph. Angot, Ch.-H. Bruneau, P. Fabrie, A penalization method to take into account obstacles in incompressible viscous flows, Numerische Mathematik 81 (1999) 497-520.

[2] A. Chorin, Numerical Solution of the Navier-Stokes Equations, Mathematics of Computation 22 (1968) 745-762.

[3] E. A. Fadlun, R. Verzicco, P. Orlandi, J. Mohd-Yusof, Combined Immersed-Boundary Finite-Difference Methods for Three-Dimensional Complex Flow Simulations, Journal of Computational Physics 161 (2000) 35-60.

[4] Th.Gallouët, L. Gastaldo, R. Herbin, J.-C. Latché, An unconditionally stable pressure correction scheme for the compressible barotropic Navier-Stokes equations, M2AN 42 (2008) 303-331.

[5] T. Ikeno, T. Kajishima, Finite-difference immersed boundary method consistent with wall conditions for incompressible turbulent flow simulations, Journal of Computational Physics 226 (2007) 1485-1508.

[6] M. Jobelin, B. Piar, Ph. Angot, J.-C. Latché, Une méthode de pénalité-projection pour les écoulements dilatables, European J. Comput. Mech. 17 (2008) 453480.

[7] J. Mohd-Yusof, Combined Immersed Boundaries/B-Splines Methods for Simulations of Flows in Complex Geometries, CTR Annual Research Briefs, NASA Ames/Stanford University, 1997.

[8] C. Peskin, Numerical Analysis of blood flow in heart, Journal of Computational Physics 25 (1977) 220-252.

[9] J. Shen, On Error Estimates of some Higher Order Projection and Penalty-Projection Methods for Navier-Stokes Equations, Numerische Mathematik. 62 (1992) 49-73.

[10] R. Temam, Sur l'approximation de la solution des équations de Navier-Stokes par la méthode des pas fractionnaires, Arch.Rational Mech. Anal. 32 (1969) 135-153.

[11] http://www-trio-u.cea.fr 